\title{
Human Hippocampal and Parahippocampal Activity during Visual Associative Recognition Memory for Spatial and Nonspatial Stimulus Configurations
}

\author{
Emrah Düzel, ${ }^{1}$ Reza Habib, ${ }^{2}$ Michael Rotte, ${ }^{1}$ Sebastian Guderian, ${ }^{1}$ Endel Tulving, ${ }^{2}$ and Hans-Jochen Heinze ${ }^{1}$ \\ ${ }^{1}$ Department of Neurology II, Otto von Guericke University, Magdeburg 39120, Germany, and ${ }^{2}$ Rotman Research Institute, Baycrest Centre, Toronto, \\ Ontario, M6A 2E1, Canada
}

\begin{abstract}
Evidence from animal studies points to the importance of the parahippocampal region (PHR) [including entorhinal, perirhinal, and parahippocampal (PHC) cortices] for recognition of visual stimuli. Recent findings in animals suggest that PHR may also be involved in visual associative recognition memory for configurations of stimuli. Thus far, however, such involvement has not been demonstrated in humans. In fact, it has been argued that associative recognition in humans is critically dependent on the hippocampal formation (HF). To better understand the division of function between HF and PHR during recognition memory in humans, we measured the activity of both areas in healthy young adults during an associative recognition memory task using functional magnetic resonance imaging. To more precisely characterize the nature of the associations that might be coded by the HF and PHR during recognition, subjects were required to learn and were later tested for associations based on either the spatial arrangements of two stimuli or the identity of two stimuli (a face and a tool). An area in the PHC was found to be more active for recognized old configurations than new configurations in both the spatial and identity conditions. The HF, on the other hand, was more active for recognition of new configurations than old configurations and also more active in the spatial than the identity condition. These data highlight the involvement of PHR in the long-term coding of associative relationships between stimuli and help to clarify the nature of its functional distinction from the HF.
\end{abstract}

Key words: associative recognition; space; object; fMRI; perirhinal cortex; parahippocampal cortex; hippocampus; novelty; familiarity; parahippocampal gyrus

\section{Introduction}

The conscious discrimination of novel events from familiar events, or recognition memory, is an important cognitive function. Ideas about the basic functional organization of medial temporal lobe (MTL) structures in recognition memory are still controversial. Conflicting results have been published as to whether the hippocampal formation (HF) (hippocampus proper, subiculum, and presubiculum) is necessary for recognition memory and, if so, whether it acts together with the parahippocampal region [perirhinal cortex (PRC), entorhinal cortex (EC), and parahippocampal cortex (PHC)] to serve a unitary function (Manns and Squire, 1999; Stark and Squire, 2001) or whether it serves a qualitatively different role (Vargha-Khadem et al., 1997; Eldridge et al., 2000; Duzel et al., 2001).

A central question (Brown and Aggleton, 2001; Stark and Squire, 2001) is whether a distinction between single-item and associative recognition is relevant to the division of function

\footnotetext{
Received May 5, 2003; revised July 29, 2003; accepted Aug. 1, 2003.

This study was supported by grants from the Deutsche Forschungsgemeinschaft (DFG/SFB 426, TP D1). R.H. is supported by a McDonnell foundation investigator initiated grant to A. R. McIntosh and a Tanenbaum postdoctoral fellowship. This study was performed as part of a Human Frontiers Science Program Organization short-term fellowship to R.H. We thank Dr. Schiltz for providing the stimulus material.

Correspondence should be addressed to Emrah Düzel, Klinik für Neurologie ll, Otto von Guericke Universität Magdeburg, Leipziger Strasse 44, 39120 Magdeburg, Germany. E-mail: emrah.dueze@@medizin.uni-magdeburg.de.

Copyright $\odot 2003$ Society for Neuroscience $\quad 0270-6474 / 03 / 239439-06 \$ 15.00 / 0$
}

within the MTL. The HF might be critical if recognition memory requires the retrieval of associative information but might not be necessary to discriminate individual items on the basis of familiarity for which the PHR, notably the PRC, seems to be critical (Cohen and Eichenbaum, 1995; Mishkin et al., 1997; Murray and Mishkin, 1998; Brown and Aggleton, 2001). Using functional magnetic resonance imaging (fMRI) in humans, some studies have reported higher activity in the HF for associative recognition over single-item recognition (Gabrieli et al., 1997; Yonelinas et al., 2001), although others have observed an equal contribution of this structure to both forms of recognition (Stark and Squire, 2000, 2001). The role of the human PHR in associative recognition is less studied, although a number of animal studies have suggested that the PRC (Messinger et al., 2001; Naya et al., 2003) and parahippocampal cortex (Malkova and Mishkin, 2003) in monkeys as well as the postrhinal cortex in rodents [thought to be analogous to primate PHC (Burwell et al., 1995)] can code different forms of associations between two items, even across modalities (Sakai et al., 1994; Murray and Bussey, 1999; Wan et al., 1999; Naya et al., 2001). In fact, the PRC is involved in the perceptual discrimination of complex objects (Buckley et al., 2001), especially when these are composed of ambiguous feature combinations (Bussey et al., 2002).

We investigated the division of labor between HF and PHR during associative recognition. Subjects distinguished between 
visually presented old configurations of items (pairs of items studied together) and new configurations of items (pairs of items studied as part of different configurations). The task could only be solved through the associative relationship between individual items because all items had been seen previously and were equally familiar. We also investigated whether the division of labor between HF and PHR is different during spatial and nonspatial associative recognition. Subjects learned (over five repetitions) the association between a face and the location of an object and, in a different session, the association between a face and the identity of an object. After learning, a recognition memory test was administered in which learned (old) and rearranged (new) spatial and nonspatial stimulus configurations had to be discriminated.

\section{Materials and Methods}

Subjects. Fifteen volunteers were paid for their participation. Because of technical difficulties involving stimulus presentation and data acquisition, data from 11 subjects (seven females; all right-handed according to self-report; mean $\pm \mathrm{SD}$ age, $24.6 \pm 2.6$ years) will be reported here. The study was approved by the ethics committee of Otto von Guericke University (Magdeburg, Germany).

Stimulus display. Stimuli consisted of pairings of gray scale faces of people (head and shoulders looking straight ahead) with cartoon drawings of one of four tools (hammer, saw, screwdriver, and wrench). Seventy such face-tool configurations were randomly assigned to each of two task conditions for each subject.

Design and procedure. The experiment consisted of a $2 \times 2$ repeatedmeasures factorial design. The first independent variable was the task, defined in terms of the nature of associative information being learned and recognized (face-identity and face-location). The second independent variable was the previous experimental history (old-new) of the test stimuli. The experiment was performed across $2 \mathrm{~d}$. On each day, subjects studied and were later tested for only one type of associative information, the order being counterbalanced across subjects.

On a given day, subjects studied, over five trials, the association between 10 faces and either the identity (face-identity condition) or spatial location (face-location condition) of one of four tools. During the study phase, each face was presented for $4 \mathrm{sec}$ in the center of a computer screen. Three seconds after the onset of face presentation, a tool appeared in one of the four corner positions of the screen and remained there for 1 sec. This was followed by a $1 \mathrm{sec}$ interstimulus interval (ISI), during which the stimuli were replaced with a fixation cross in the center of the screen. On each trial, subjects were required to indicate the identity or location of the tool before its appearance (after $3 \mathrm{sec}$ ) by pressing one of four buttons (because subjects had not seen the pairings of the faces and identities or locations of the objects on the first trial, they were instructed to guess and move their thumb). The face-location associations that needed to be learned and then discriminated during recognition could be best described as egocentric (personal reference frame) rather than allocentric (extrapersonal reference frame) (Holdstock et al., 2000). The reason why faces and tools were not presented simultaneously with the exception of the $1 \mathrm{sec}$ feedback period was to encourage the encoding of the faces and the tools or their locations as separate pieces of information that needed to be associated rather than components of a single compound stimulus (Erickson and Desimone, 1999).

After the fifth study trial, subjects received an associative yes-no recognition test. New test stimuli ("new configurations") in the face-identity task consisted of a studied face paired with one of the three tools that was not paired with it during study, presented in the same location as the original tool. New test stimuli in the face-location task consisted of a studied face presented with its paired tool but in a new location. Figure 1 depicts these two possible scenarios. Subjects were required to discriminate new stimulus configurations from old stimulus configurations. Note that the new test configurations consisted of highly familiar component parts: a previously viewed face and previously viewed tools. These stimuli were new only in the sense that an association between the face and the identity or location of the tool had not been established previ-

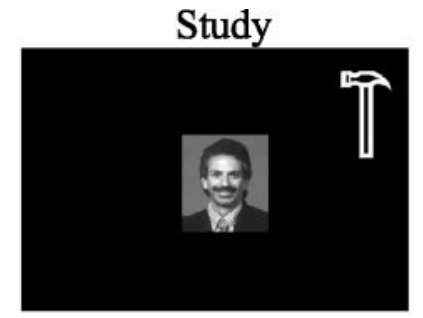

Face -Identity

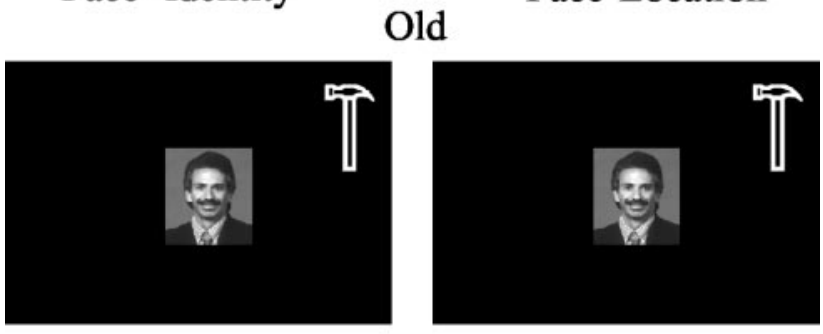

New
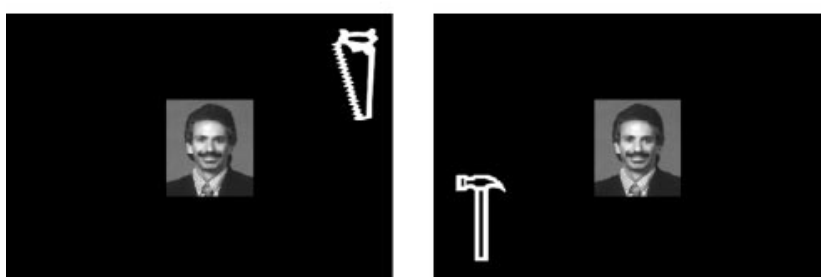

Figure 1. This figure depicts the experimental paradigm. At study, subjects view a face paired with an object ( 1 of 4 tools) in one of the four corner screen positions. In the face-identity condition, subjects are instructed to learn the association between the face and the identity of the object. In the face-location condition, subjects are instructed to learn the association between the face and the spatial location of the object. Learning occurs over five trials. At test, subjects see either the original configuration or new configurations of the familiar stimuli. In the face-identity condition, new configurations consist of the original face with a new tool in the position of the original tool. In the face-location condition, new configurations consist of the original face with the original tool in a new spatial location. Subjects are required to discriminate the new configurations from the familiar configurations by pressing a response key.

ously. Furthermore, the nature of the old test configurations was the same in both task conditions; it was only the task and hence the subject's judgment (concerning the spatial location or object identity) that differed. Each face-tool configuration was presented for $3 \mathrm{sec}$ with a $12 \mathrm{sec}$ ISI to permit the blood oxygenation level-dependent (BOLD) response to return to baseline. During each recognition test, five old configurations were randomly intermixed with five new configurations.

This learning and recognition procedure was repeated seven times, each time with a different set of 10 face-tool and face-location configurations.

Scanning and analysis methods. AMRI imaging was performed during the recognition test with a GE 1.5 T Signa Neurovascular system using a standard quadrature head coil. Visual images were back projected onto a screen that the subject could see through a mirror. Subject responses were given by magnet compatible buttons. Conventional high-resolution structural images were acquired before functional imaging (T1 weighted radio frequency-spoiled gradient-recalled acquisition in the steady state sequence). Each functional run during the recognition test consisted of 54 continuous whole-brain volumes $\left(\mathrm{T} 2{ }^{\star}\right.$ echo planar gradient echo sequence; repetition time, $3 \mathrm{sec}$; echo time, $40 \mathrm{msec} ; 30 \times 5$ gap $1 \mathrm{~mm}$ slices perpendicular to the hippocampal axis; field of view, $20 \mathrm{~mm}$; matrix size, $64 \times 64$; voxel size, $3.13 \times 3.13 \times 6 \mathrm{~mm}$ ). Each subject except one (who contributed six) contributed seven study-test runs to the data set. Six participants also had high-resolution T1-weighted MR images (124 sagittal slices; three-dimensional spoiled gradient-recalled acquisition in a 
steady state). The images of these six participants were normalized (voxel size, $1 \times 1 \times 1 \mathrm{~mm}^{2}$ ), and a mean image of the normalized images was created and smoothed using a Gaussian kernel of $2 \times 2-2 \mathrm{~mm}$. This mean image was used to visualize the fMRI results.

Analysis was performed with SPM99 software (Wellcome Trust, London, UK) after discarding the first four volumes to allow for stabilization of the BOLD signal. Each subject's functional volumes were (1) corrected for differences in the acquisition times of different slices attributable to the interleaving of the slices, (2) realigned to their first scan to correct for movement, (3) spatially normalized to an echo planar imaging template [Montreal Neurological Institute (MNI) reference brain; voxels were resized to $2 \times 2 \times 4 \mathrm{~mm}$ ), and (4) spatially smoothed ( $4 \mathrm{~mm}$ Gaussian kernel). Identification of medial temporal lobe structures, notably the EC, the PRC, and the PHC, was based on the description of Pruessner et al. (2002). Accordingly, EC, PRC, and PHC are distributed along the medial and lateral bank of the collateral sulcus. The PRC surrounds the $\mathrm{EC}$ anteriorly and laterally and posteriorly. Posterior to the PRC, the PHC continues to a level with the posterior hippocampus. The border between the posterior PRC and the PHC lies $\sim 5 \mathrm{~mm}$ posterior to the disappearance of the intralimbic gyrus.

Statistical modeling for event-related design included all conditions of interest (old and new stimulus configurations) using a canonical hemodynamic response function for all event types. Subject-specific contrasts were estimated using a fixed-effects model. For averaging across subjects, a second-level analysis was performed using the individual contrasts in a random-effects model. Results are reported in MNI coordinate system. Because of our a priori hypotheses about the role of the MTL during associative recognition, a level of $p<0.005$ uncorrected for multiple comparisons was chosen for the activation threshold.

\section{Results}

\section{Behavioral results}

Hit rates, false alarm rates (FA), and corrected recognition (CR) performance (hit - false alarm rates) were virtually identical in the face-identity (hits, 0.93; FA, 0.05; CR, 0.88) and face-location (hits, 0.94; FA, 0.06; CR, 0.88) conditions. Reaction times to correct recognition decisions (hits and correct rejections) in the face-location and face-identity tasks were submitted to a twoway repeated-measures ANOVA. Subjects responded more quickly in the face-location task $(1721.81 \mathrm{msec})$ than in the faceidentity task $\left(1962.79 \mathrm{msec} ; F_{(1,9)}=13.42 ; p<0.01\right)$ but neither the difference between hits $(1820.34 \mathrm{msec})$ and correct rejections (1864.25 msec) nor the two-way interaction reached significance.

\section{fMRI}

Differential activity within the MTL was noted in three comparisons: (1) face-location - face-identity, (2) new - old, and (3) old - new

Activity in right hippocampus $(x, y, z=30,14,16$; $z=3.39$; $p<0.001$; activated volume, $48 \mathrm{~mm}^{3}$ ) was greater when subjects made recognition decisions during the face-location task than when subjects made recognition decisions during the face-identity task, regardless of whether the configurations were new or old (Fig. 2). No comparable MTL activity was noted when the opposite subtraction (face-identity - face-location) was performed.

When the recognition of new configurations was compared with the recognition of old configurations, regardless of the relevant task dimension (face-identity and face-location), greater activity was noted in the right anterior hippocampus $(x, y, z=24$, 14,$16 ; Z=2.82 ; p=0.002$; activated volume, $32 \mathrm{~mm}^{3}$ ) (Fig. 3). When the opposite contrast was performed (old configurations - new configurations), greater activity was noted posteriorly, in the fundus of the right collateral sulcus, a region that corresponds to the PHC and that possibly includes portions of the posterior $\operatorname{PRC}(x, y, z=36,34,16 ; Z=2.82 ; p=0.002$; activated volume, $112 \mathrm{~mm}^{3}$ ) (Fig. 4).
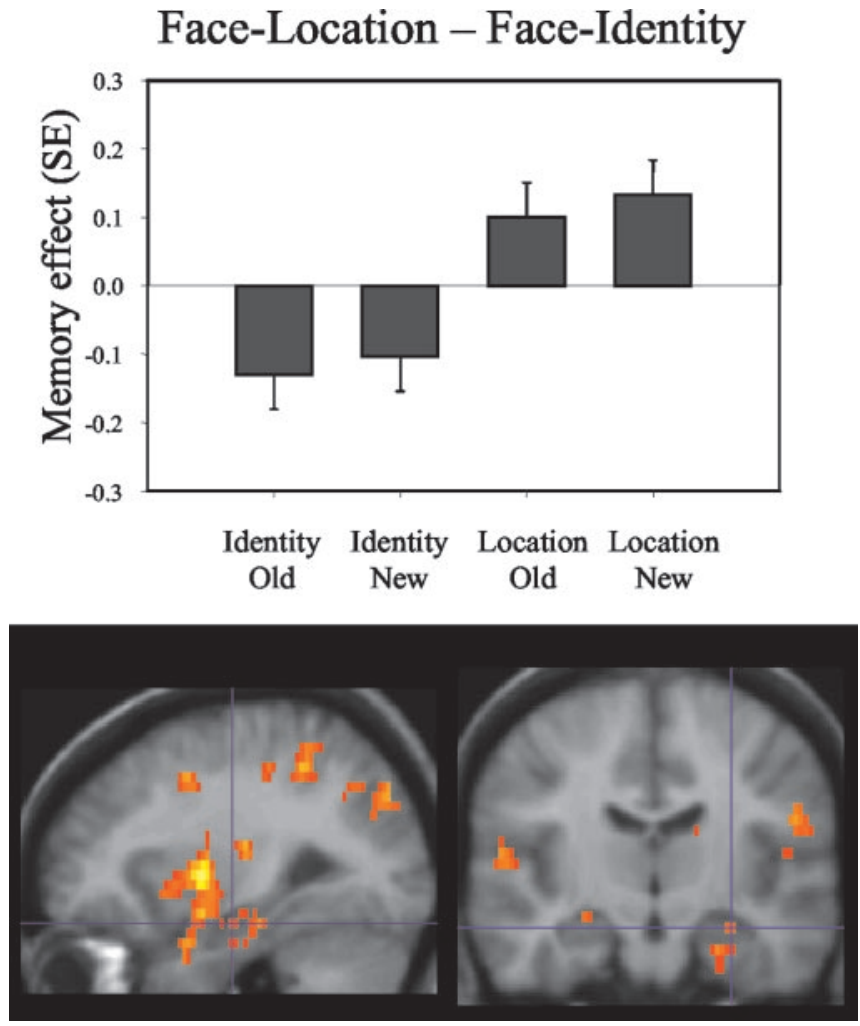

Figure 2. This figure indicates that recognition in the face-location (Location) condition elicited higher activations in bilateral hippocampus than recognition in the face-identity (Identity) condition. Bar plots show the contrast (Memory effect) of parameter estimates (betas, from the peak voxel) for old and new configurations in both conditions. As in Figures 3 and 4, the activations are displayed on averaged structural images of six participants.

We submitted these data to a 2 region (HF and PHC-PRC) $\times$ 2 task (face-identity and face-location) $\times 2$ history (new and old) repeated-measures ANOVA. The two-way interaction between region and history was significant $\left(F_{(1,8)}=49.98 ; p<\right.$ $0.001)$, indicating that, in the anterior hippocampus, activity was stronger for new configurations than old configurations regardless of the task, whereas in the PHC-PRC, activity was stronger for old configurations than new configurations regardless of the task (Fig. 5).

\section{Discussion}

In agreement with data from a number of animal studies that investigated the role of the PRC (Murray and Bussey, 1999) and PHC (Malkova and Mishkin, 2003) in memory retrieval, our results show that these regions are also responsive to the associative relationship between familiar items. An area in the PHC that possibly also included portions of the posterior PRC (hence referred to as PHC-PRC) was significantly more active for old configurations of familiar items, regardless of the nature (spatial vs nonspatial) of the associative information (Figs. 4, 5). However, unlike the commonly observed decrease in neural activity for repeated individual items (Fahy et al., 1993; Desimone, 1996; Brown and Xiang, 1998), our results demonstrated an increase in neural activity for repeated (old) configurations relative to new configurations.

There may be two reasons, besides the difference in technique, for why the PHC-PRC showed a relative increment in activity to old configurations ("repetition enhancement"; Desimone, 1996). First, in most previous recognition memory experiments in ani- 


\section{New - Old}
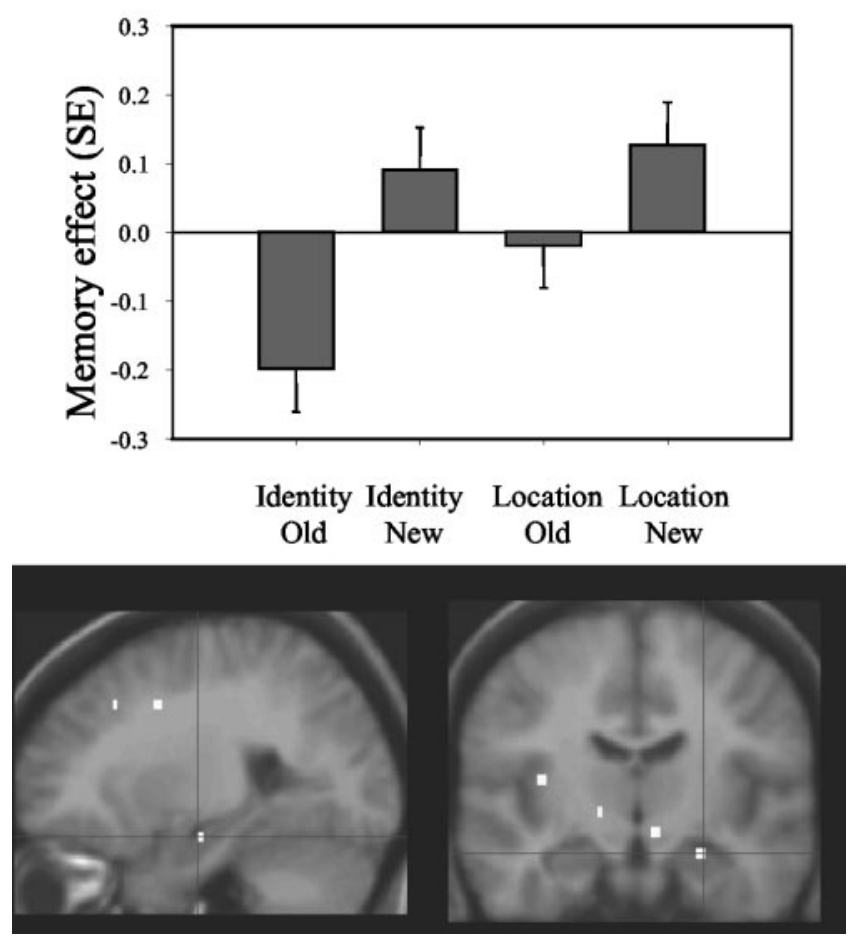

Figure 3. This figure identifies regions within the medial temporal lobes whose activity distinguishes correct rejections of new configurations from recognition of old configurations. Bar plots show the contrast (Memory effect) of parameter estimates (betas, from the peak voxel) for old and new configurations in the face-location (Location) and the face-identity (Identity) conditions. Activations in the anterior hippocampus are greater when subjects correctly reject new configurations than when they recognize old configurations.

mals and humans, new stimuli consisted of items that had not been encountered previously in the experiment, whereas in the present study, new stimuli consisted of configurations of individual items that had been encountered previously and were thus familiar. Miller and Desimone (1994) have shown that, when repeated nontarget items (item "B" in the "A-B-B-A" version of the delayed matching to sample paradigm) are intermixed with repeated target items, the traditional repetition suppression response is not observed for the repeated target items. Rather, a repetition suppression response is observed for the nontarget items ("B" items), but a repetition enhancement response is observed for the target items (" $\mathrm{A}$ " items). This observation makes good sense because, had both types of items generated a repetition-suppression response, neuronal firing would not provide a means for distinguishing between them. A higher PHC-PRC activity for old configurations in the present study is compatible with these animal data because the targets (old configurations) and distracters (new configurations) in both studies consisted of repeated items. We can therefore assume that, in our experiment too, repetition suppression could not have helped to discriminate target events from distracters.

Second, the incremental PHC-PRC activity for old configurations when compared with new configurations could reflect the activity of certain PRC neurons that have been reported to show enhanced activity to old items if these old items tag learned associations to other items (Sakai and Miyashita, 1991; Sakai et al., 1994; Jagadeesh et al., 2001; Naya et al., 2001). The activity of these "pair-coding" (Sakai and Miyashita, 1991; Sakai et al., 1994) or "target-related" (Naya et al., 2001) neurons differ from famil-

\section{Old - New}
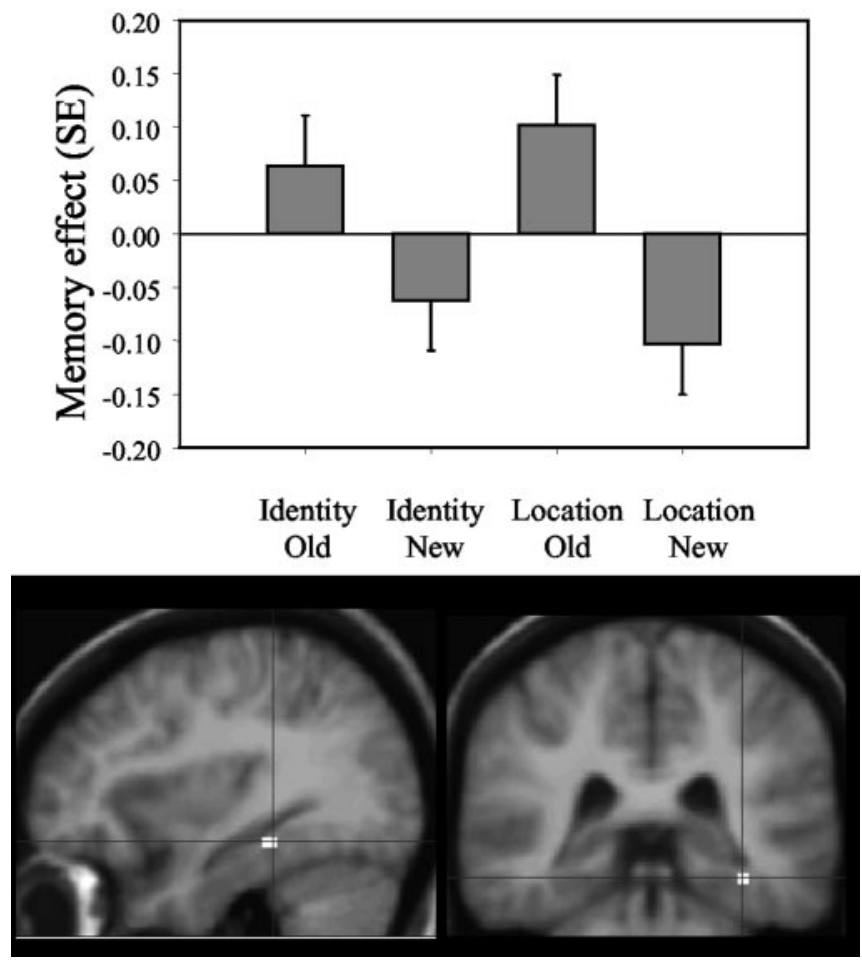

Figure 4. This figure identifies regions within the medial temporal lobes whose activity distinguishes recognition of old configurations from recognition of new configurations. Bar plots show the contrast (Memory effect) of parameter estimates (betas, from the peak voxel) for old and new configurations in the face-location (Location) and the face-identity (Identity) conditions. Activations in the parahippocampal cortex are greater when subjects recognize old configurations than when they correctly reject new configurations.

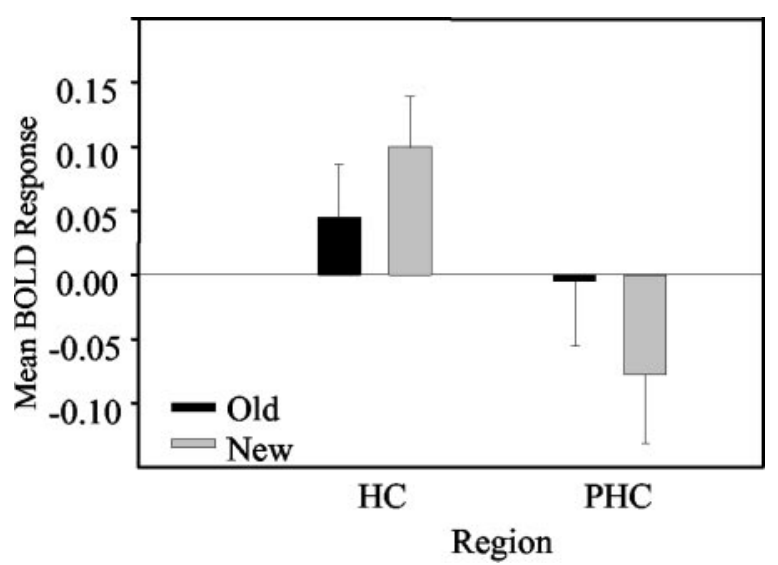

Figure 5. This figure shows the interaction between brain activity (mean of fitted BOLD responses across subjects) in the $\mathrm{PHC}$ and the hippocampus $(\mathrm{HC})$ on the one hand and the previous history (new-old) of the stimulus configurations on the other. The graph indicates that, in the hippocampus, activation is greater for new configurations than old configurations, whereas in the PHC, the opposite is true: activation is greater for old than new configurations.

iarity neurons in that they show greater activity to old items if their associative pair can be retrieved (Murray and Bussey, 1999). Although not explicitly discussed in the ensuing publications, a similar property is suggested for the PHR by hemodynamic neuroimaging studies in humans after closer inspection of published data. In a direct comparison, Yonelinas et al. (2001) found higher activity in the right parahippocampal region for old items when 
subjects retrieved the color of studied objects (associative recognition memory task) compared with just making a recognition judgment (simple recognition memory task). Furthermore, right PHR activity was reported to be higher in a task that required the report of recollective experience when subjects reported that they recollected a word as opposed to having a mere familiarity with it (Eldridge et al., 2000). Together with the present results, these animal and human neuroimaging data provide evidence that parahippocampal activity for old stimuli is higher during associative recognition than during single-item recognition.

Holding constant the familiarity of recognition test items in our study is a major departure from previous hemodynamic neuroimaging studies of recognition memory that have reported repetition decrement as a commonly observed response in the PHR (Henson et al., 2003) (for an exception, see Stark and Squire, 2001). To what extent the prevalence of repetition decrement or enhancement is contingent on the associative nature of the recognition memory task, or the presence or absence of new items as distracters, remains to be determined. Another possibility that needs to be elucidated is whether repetition responses might differ in the anterior and posterior portions of the PHR such that repetition decrement responses might be more prevalent anteriorly (Henson et al., 2003).

As in previous studies (Moscovitch et al., 1995; Burgess et al., 2001), we also observed a preference of the HF for the processing of spatial information; right anterior HF activity was greater during the face-location than the face-identity task, regardless of the previous history of the stimulus configurations (Fig. 2). However, a critical observation in our study was that of a higher right anterior HF activation for new configurations, regardless of whether the subjects' task was to recognize a face-location or a face-identity association (Fig. 3). This finding provides support for the hypothesis that there is a common hippocampal code that is necessary for spatial memory as well as for nonspatial episodic memory that has the cognitive quality of spatial or nonspatial "events" rather than of spatial coordinates (Wood et al., 1999, 2000; Eichenbaum, 2001). This notion of an hippocampal "event-code" accounts for the observation that patients with relatively selective hippocampal lesions show a marked impairment of nonspatial episodic memory (Murray and Bussey, 1999; Eldridge et al., 2000; Brown and Aggleton, 2001; Duzel et al., 2001), as well as of topographical memory (Spiers et al., 2001; Astur et al., 2002) and associative spatial memory (Holdstock et al., 2000).

At the same time, our results qualitatively distinguish $\mathrm{HF}$ activation from PHR activation. In our study, HF activation can be interpreted to be related to the detection and/or encoding of novel information, whereas PHR activation appears to be related to the retrieval of learned information. This dissociation is compatible with the proposal that HF and PHR mediate a qualitatively different form of memory process (Vargha-Khadem et al., 1997; Mishkin et al., 1998; Duzel et al., 2001). Extending previous reports (Tulving et al., 1996) (for review, see Ranganath and Rainer, 2003), the present results also show that the "novelty" of a stimulus that is being detected and/or encoded by the HF is not contingent on an item being presented for the first time in the experiment; rather, novelty can also be attributable to the rearrangement of learned configurations of items even if item information within the configurations is highly familiar (Wan et al., 1999). In fact, our results suggest that the associative novelty response found here might be special to the HF, whereas itemnovelty effects might be common to other medial temporal lobe structures that show a repetition suppression response to old items.
Two remaining issues concerning the division of function between the HF and PHR merit additional discussion. First, how is it possible that patients with relatively selective hippocampal injury are capable of acquiring associative information (VarghaKhadem et al., 1997; Murray and Bussey, 1999; Baddeley et al., 2001) although their episodic memory is severely impaired? It can argued that the PHR, which is apparently intact in these patients, should be involved in such acquisition. This argument is supported by preserved object-place associative learning in monkeys with neurotoxic hippocampal lesions (Malkova and Mishkin, 2003) but the impairment of such learning after PHC lesions. Furthermore, the perceptual role of the PHR in the discrimination of objects composed of conjunctions of ambiguous features (Bussey et al., 2003) support its putative role in associative learning. However, to what extent the repetition enhancement response observed here during recognition can contribute to learning of associative information in the PHR remains to be determined. A second issue is the flexibility of associative representations in the $\mathrm{HF}$ and $\mathrm{PRH}$ (Cohen and Eichenbaum, 1995). Rather than being confined to a rigid relationship between sequences of events or pairs of stimuli, the HF seems to allow flexible access to these relationships, a property that has been termed transitivity (Dusek and Eichenbaum, 1997), and it remains to be determined to what extent this property is applicable also to the PRH.

To summarize, the present data show that the division of function between the HF and the PHR need not be limited to a distinction between associative (HF) and nonassociative (PHR) memory. Rather, both of these regions can represent associative information necessary to discriminate configurations of items on the basis of the history of their previous occurrence. Furthermore, when memory has to rely solely on associative information, that is, when individual items are equally familiar, the usual response pattern of PHR areas, namely a decrease in neural response to the learned stimuli, is reversed to an increase in neural response. Finally, the response pattern of the two regions suggests a qualitatively different role for them, with the HF more involved in the detection and/or encoding of novel configurations and the PHR more involved in the recognition of the learned configurations.

\section{References}

Astur RS, Taylor LB, Mamelak AN, Philpott L, Sutherland RJ (2002) Humans with hippocampus damage display severe spatial memory impairments in a virtual Morris water task. Behav Brain Res 132:77-84.

Baddeley A, Vargha-Khadem F, Mishkin M (2001) Preserved recognition in a case of developmental amnesia: implications for the acquisition of semantic memory? J Cognit Neurosci 13:357-369.

Brown M, Aggleton JP (2001) Recognition memory: what are the roles of the perirhinal cortex and the hippocampus. Nat Rev Neurosci 2:51-61.

Brown MW, Xiang JZ (1998) Recognition memory: neuronal substrates of the judgement of prior occurrence. Prog Neurobiol 55:149-189.

Buckley MJ, Booth MC, Rolls ET, Gaffan D (2001) Selective perceptual impairments after perirhinal cortex ablation. J Neurosci 21:9824-9836.

Burgess N, Maguire EA, Spiers HJ, O’Keefe J (2001) A temporoparietal and prefrontal network for retrieving the spatial context of lifelike events. NeuroImage 14:439-453.

Burwell RD, Witter MP, Amaral DG (1995) Perirhinal and postrhinal cortices of the rat: a review of the neuroanatomical literature and comparison with findings from the monkey brain. Hippocampus 5:390-408.

Bussey TJ, Saksida LM, Murray EA (2002) Perirhinal cortex resolves feature ambiguity in complex visual discriminations. Eur J Neurosci 15:365-374.

Bussey TJ, Saksida LM, Murray EA (2003) Impairments in visual discrimination after perirhinal cortex lesions: testing "declarative" vs. "perceptual-mnemonic" views of perirhinal cortex function. Eur J Neurosci 17:649-660. 
Cohen NJ, Eichenbaum H (1995) Memory, amnesia and the hippocampal system. Cambrigde, MA: MIT.

Desimone R (1996) Neural mechanisms for visual memory and their role in attention. Proc Natl Acad Sci USA 93:13494-13499.

Dusek JA, Eichenbaum H (1997) The hippocampus and memory for orderly stimulus relations. Proc Natl Acad Sci USA 94:7109-7114.

Duzel E, Vargha-Khadem F, Heinze HJ, Mishkin M (2001) Brain activity evidence for recognition without recollection after early hippocampal damage. Proc Natl Acad Sci USA 98:8101-8106.

Eichenbaum H (2001) The hippocampus and declarative memory: cognitive mechanisms and neural codes. Behav Brain Res 127:199-207.

Eldridge LL, Knowlton BJ, Furmanski CS, Bookheimer SY, Engel SA (2000) Remembering episodes: a selective role for the hippocampus during retrieval. Nat Neurosci 3:1149-1152.

Erickson CA, Desimone R (1999) Responses of macaque perirhinal neurons during and after visual stimulus association learning. J Neurosci 19:10404-10416.

Fahy FL, Riches IP, Brown MW (1993) Neuronal signals of importance to the performance of visual recognition memory tasks: evidence from recordings of single neurones in the medial thalamus of primates. Prog Brain Res 95:401-416.

Gabrieli JDE, Brewer JB, Desmond JE, Glover GH (1997) Separate neural bases of two fundamental memory processes in the human medial temporal lobe. Science 276:264-266.

Henson RN, Cansino S, Herron JE, Robb WG, Rugg MD (2003) A familiarity signal in human anterior medial temporal cortex? Hippocampus 13:301-304.

Holdstock JS, Mayes AR, Cezayirli E, Isaac CL, Aggleton JP, Roberts N (2000) A comparison of egocentric and allocentric spatial memory in a patient with selective hippocampal damage. Neuropsychologia 38:410-425.

Jagadeesh B, Chelazzi L, Mishkin M, Desimone R (2001) Learning increases stimulus salience in anterior inferior temporal cortex of the macaque. J Neurophysiol 86:290-303.

Malkova L, Mishkin M (2003) One-trial memory for object-place associations after separate lesions of hippocampus and posterior parahippocampal region in the monkey. J Neurosci 23:1956-1965.

Manns JR, Squire LR (1999) Impaired recognition memory on the Doors and People Test after damage limited to the hippocampal region. Hippocampus 9:495-499.

Messinger A, Squire LR, Zola SM, Albright TD (2001) Neuronal representations of stimulus associations develop in the temporal lobe during learning. Proc Natl Acad Sci USA 98:12239-12244.

Miller EK, Desimone R (1994) Parallel neuronal mechanisms for shortterm memory. Science 263:520-522.

Mishkin M, Suzuki WA, Gadian DG, Vargha-Khadem F (1997) Hierarchical organization of cognitive memory. Philos Trans R Soc Lond B Biol Sci 352:1461-1467.

Mishkin M, Vargha-Khadem F, Gadian DG (1998) Amnesia and the organization of the hippocampal system. Hippocampus 8:212-216.

Moscovitch M, Kapur S, Kohler S, Houle S (1995) Distinct neural correlates of visual long-term memory for spatial location and object identity: a positron emission tomography study in humans. Proc Natl Acad Sci USA 92:3721-3725.

Murray EA, Bussey TJ (1999) Perceptual-mnemonic functions of the perirhinal cortex. Trends Cogn Sci 3:142-151.

Murray EA, Mishkin M (1998) Object recognition and location memory in monkeys with excitotoxic lesions of the amygdala and hippocampus. J Neurosci 18:6568-6582.

Naya Y, Yoshida M, Miyashita Y (2001) Backward spreading of memoryretrieval signal in the primate temporal cortex. Science 291:661-664.

Naya Y, Yoshida M, Miyashita Y (2003) Forward processing of long-term associative memory in monkey inferotemporal cortex. J Neurosci 23:2861-2871.

Pruessner JC, Kohler S, Crane J, Pruessner M, Lord C, Byrne A, Kabani N, Collins DL, Evans AC (2002) Volumetry of temporopolar, perirhinal, entorhinal and parahippocampal cortex from high-resolution MR images: considering the variability of the collateral sulcus. Cereb Cortex 12:1342-1353.

Ranganath C, Rainer G (2003) Neural mechanisms for detecting and remembering novel events. Nat Rev Neurosci 4:193-202.

Sakai K, Miyashita Y (1991) Neural organization for the long-term memory of paired associates. Nature 354:152-155.

Sakai K, Naya Y, Miyashita Y (1994) Neuronal tuning and associative mechanisms in form representation. Learn Mem 1:83-105.

Spiers HJ, Burgess N, Hartley T, Vargha-Khadem F, O’Keefe J (2001) Bilateral hippocampal pathology impairs topographical and episodic memory but not visual pattern matching. Hippocampus 11:715-725.

Stark CE, Squire LR (2000) fMRI activity in the medial temporal lobe during recognition memory as a function of study-test interval. Hippocampus 10:329-337.

Stark CE, Squire LR (2001) Simple and associative recognition memory in the hippocampal region. Learn Mem 8:190-197.

Tulving E, Markowitsch HJ, Craik FE, Habib R, Houle S (1996) Novelty and familiarity activations in PET studies of memory encoding and retrieval. Cereb Cortex 6:71-79.

Vargha-Khadem F, Gadian DG, Watkins KE, Connelly A, Van Paesschen W, Mishkin M (1997) Differential effects of early hippocampal pathology on episodic and semantic memory. Science 277:376-380.

Wan H, Aggleton JP, Brown MW (1999) Different contributions of the hippocampus and perirhinal cortex to recognition memory. J Neurosci 19:1142-1148.

Wood ER, Dudchenko PA, Eichenbaum H (1999) The global record of memory in hippocampal neuronal activity. Nature 397:613-616.

Wood ER, Dudchenko PA, Robitsek RJ, Eichenbaum H (2000) Hippocampal neurons encode information about different types of memory episodes occurring in the same location. Neuron 27:623-633.

Yonelinas AP, Hopfinger JB, Buonocore MH, Kroll NE, Baynes K (2001) Hippocampal, parahippocampal and occipital-temporal contributions to associative and item recognition memory: an fMRI study. NeuroReport $12: 359-363$. 